\title{
A Hemodynamically Significant Patent Ductus Arteriosus Does Not Affect Cerebral or Renal Tissue Oxygenation in Preterm Infants
}

\author{
Michelle E. van der Laan ${ }^{a}$ Marcus T.R. Roofthooft ${ }^{b}$ Marian W.A. Fries ${ }^{b}$ \\ Rolf M.F. Berger ${ }^{b}$ Trijntje E. Schat ${ }^{a}$ Anne G.J.F. van Zoonen ${ }^{a}$ Jozien C. Tanis ${ }^{a}$ \\ Arend F. Bos ${ }^{a}$ Elisabeth M.W. Kooi ${ }^{a}$ \\ a Division of Neonatology, and ${ }^{\mathrm{b}}$ Department of Pediatric Cardiology, Center for Congenital Heart Diseases, Beatrix \\ Children's Hospital, University Medical Center Groningen, University of Groningen, Groningen, The Netherlands
}

\section{Key Words}

Near-infrared spectroscopy · Echocardiography · Patent ductus arteriosus . Preterm infants - Tissue oxygenation

\begin{abstract}
Background: Patent ductus arteriosus (PDA) is common in preterm infants and is associated with significant morbidity. To determine whether the PDA is hemodynamically significant (HSDA), several echocardiographic parameters have been suggested, including retrograde diastolic blood flow in the descending aorta (Dao). Objective: To assess the impact of an HSDA, including retrograde diastolic flow in the Dao, on regional tissue oxygen saturation $\left(\mathrm{rSO}_{2}\right)$ and extraction measured by near-infrared spectroscopy (NIRS). Methods: This is a prospective observational cohort study in which we included preterm infants ( $G A<32$ weeks) who underwent echocardiographic screening because of clinical signs of an HSDA within 2 weeks after birth. We measured cerebral and renal $\mathrm{rSO}_{2}$ on the day of echocardiography. HSDA was diagnosed if left-to-right shunting through the PDA was accompanied by left atrial-to-aortic root ratio $>1.4$ and/or left pulmonary artery end-diastolic flow velocity $>0.2 \mathrm{~m} / \mathrm{s}$ and/or retrograde diastolic blood flow in the
\end{abstract}

\section{KARGER}

E-Mail karger@karger.com www.karger.com/neo

\section{C) 2016 The Author(s) \\ Published by S. Karger AG, Basel 1661-7800/16/1102-0141\$39.50/0}

This article is licensed under the Creative Commons AttributionNonCommercial-NoDerivatives 4.0 International License (CC BYNC-ND) (http://www.karger.com/Services/OpenAccessLicense) Usage and distribution for commercial purposes as well as any distribution of modified material requires written permission.
Dao. Results: Forty-nine infants were included, with a median GA of 27.6 weeks (IQR: 26.1-29.0), birth weight of $980 \mathrm{~g}$ (IQR: 800-1,200), and postnatal age of $77 \mathrm{~h}$ (IQR: 70 107). Infants with a closed duct $(n=11)$, a non-HSDA ( $n=$ $18)$, and an HSDA ( $n=20)$ had similar cerebral and renal NIRS measurements. Retrograde diastolic blood flow in the Dao, present in 11 infants with PDA, also did not affect cerebral and renal NIRS measurements. Conclusion: In preterm infants with clinical signs of an HSDA within 2 weeks after birth, cerebral and renal oxygen saturation and extraction are not affected by an HSDA or by retrograde diastolic blood flow in the Dao.

(C) 2016 The Author(s)

Published by S. Karger AG, Basel

\section{Introduction}

Patent ductus arteriosus (PDA) is a common finding in preterm infants. In the presence of a large PDA and decreasing pulmonary-to-systemic vascular resistance ratio, significant systemic to pulmonary shunting occurs, which results in pulmonary hyperperfusion and systemic hypoperfusion. Although causality has not yet been proven sufficiently, persistent patency of the ductus arteriosus 
(DA) in preterm infants has been associated with significant morbidities such as intraventricular hemorrhage, necrotizing enterocolitis, and renal insufficiency, possibly due to decreased systemic blood flow resulting in tissue ischemia [1-4].

Whether a PDA will impact organ tissue oxygen supply may depend on its hemodynamic significance. However, no consensus exists concerning the echocardiographic definition of a hemodynamically significant PDA (HSDA). The size of the DA and the direction of shunting through the DA have been used as treatment criteria but may not accurately quantify the transductal shunt. Echocardiographic parameters believed to better reflect the circulatory consequences of an HSDA include the presence of increased antegrade diastolic blood flow in the left pulmonary artery (LPA), an increased left atrial-to-aortic root (LA:Ao) ratio, and the presence of ductal steal associated with retrograde diastolic blood flow in the descending aorta (Dao) [5]. Previous studies have demonstrated that these parameters reflect large transductal shunt volumes $[6,7]$. Whether such shunt leads to decreased pre- or postductal organ tissue oxygen supply in preterm infants remains unclear.

Near-infrared spectroscopy (NIRS) is a noninvasive tool with which the regional tissue oxygen saturation $\left(\mathrm{rSO}_{2}\right)$ of the underlying tissue can be measured. When transcutaneous arterial oxygen saturation $\left(\mathrm{spO}_{2}\right)$ is measured simultaneously, fractional tissue oxygen extraction (FTOE) can be calculated, which reflects the balance between tissue oxygen delivery and tissue oxygen consumption [8]. In the presence of constant tissue oxygen metabolism, FTOE reflects tissue perfusion.

Previous studies using NIRS to assess organ oxygenation in preterm infants with PDA used different criteria for the determination of an HSDA and found conflicting results concerning the impact of PDA on pre- and postductal organ oxygenation [9-12]. The specific impact of echocardiographic parameters of hemodynamic significance of a PDA on regional tissue oxygen supply has not been investigated previously.

Our first aim, therefore, was to assess whether upper and lower organ tissue oxygen delivery, as reflected by cerebral and renal $\mathrm{rSO}_{2}$ and FTOE, is affected in infants with an HSDA compared with infants with a non-HSDA and infants with a closed DA. Our second aim was to assess the impact of separate echocardiographic parameters of supposed hemodynamic significance, particularly retrograde diastolic blood flow in the Dao as a marker for ductal steal, on cerebral and renal $\mathrm{rSO}_{2}$ and FTOE in infants with PDA.

\section{Methods}

\section{Patients}

We included patients from two simultaneous prospective observational cohort studies conducted at the neonatal intensive care unit of University Medical Center Groningen between February 2012 and March 2014. In both, preterm infants (GA <32 weeks) who underwent echocardiographic screening for a PDA during the first 2 weeks after birth were eligible for inclusion. Infants with cardiovascular defects other than a PDA, with other major congenital defects or with persistent pulmonary hypertension of the newborn were excluded. Depending on the study in which the patient was included, NIRS was measured either continuously or for $2 \mathrm{~h}$ daily. The studies were approved by the ethics review board of University Medical Center Groningen. Written informed parental consent was obtained in all cases.

\section{Echocardiography}

Screening echocardiography was routinely performed in all infants who still required mechanical ventilation or continuous positive airway pressure on the third postnatal day. Furthermore, echocardiography was performed when an HSDA was otherwise suspected clinically (continuing respiratory distress, unexplained need for supplemental oxygen, metabolic acidosis, bounding pulses and/or increased pulse pressure). Echocardiograms were made by the attending pediatric cardiologist or a trained pediatric ultrasonographist, using a Vivid-I ultrasound scanner (GE Healthcare, $\mathrm{UK})$ with a $10-\mathrm{MHz}$ probe.

A PDA was classified as hemodynamically significant when a DA was present with predominantly left-to-right shunting across the DA associated with (1) LA:Ao ratio $>1.4$ and/or (2) end-diastolic blood flow velocity in the LPA $>0.2 \mathrm{~m} / \mathrm{s}$ and/or (3) diastolic flow reversal in the Dao.

Ductal flow recordings were made from a high parasternal view using continuous-wave Doppler. The LA:Ao ratio was measured using M-Mode pictures from the parasternal long axis view using the leading-edge-to-leading-edge technique. End-diastolic flow velocity in the LPA and postductal flow velocity in the Dao were measured using pulsed-wave Doppler from a high parasternal view. Furthermore, we measured the internal ductal diameter, the left ventricular end-diastolic diameter, fractional shortening, and ejection fraction. The ductal diameter was measured during endsystole at the point of maximum constriction using color Doppler flow, with care taken to minimalize color gain to avoid color interference outside of the vessel wall. The left ventricular end-diastolic diameter, fractional shortening, and ejection fraction were measured using M-Mode pictures from the parasternal long axis view. Echocardiograms were stored for off-line analysis and were analyzed as a batch by a trained researcher (M.E.v.d.L.) who was blinded to the NIRS data.

\section{Multisite NIRS Measurements}

We used the INVOS 5100C near-infrared spectrometer and neonatal SomaSensors (Covidien, Mansfield, Mass., USA) to measure cerebral and renal $\mathrm{rSO}_{2}\left(\mathrm{r}_{\mathrm{c}} \mathrm{SO}_{2}\right.$ and $\left.\mathrm{r}_{\mathrm{r}} \mathrm{SO}_{2}\right)$ on the day of echocardiography. Sensors were placed on the frontoparietal side of the infant's head $\left(\mathrm{r}_{\mathrm{c}} \mathrm{SO}_{2}\right)$ and on the lateral posterior flank $\left(\mathrm{r}_{\mathrm{r}} \mathrm{SO}_{2}\right)$.

NIRS data were stored for off-line analysis. We calculated FTOE for each location as: FTOE $=\left(\mathrm{spO}_{2}-\mathrm{rSO}_{2}\right) / \mathrm{spO}_{2}$. As ductal steal may preferentially occur from the postductal circulation [7, 
Table 1. Demographic, clinical and echocardiographic characteristics of infants with a closed DA, a non-HSDA and an HSDA, and NIRS and $\mathrm{spO}_{2}$ measurements

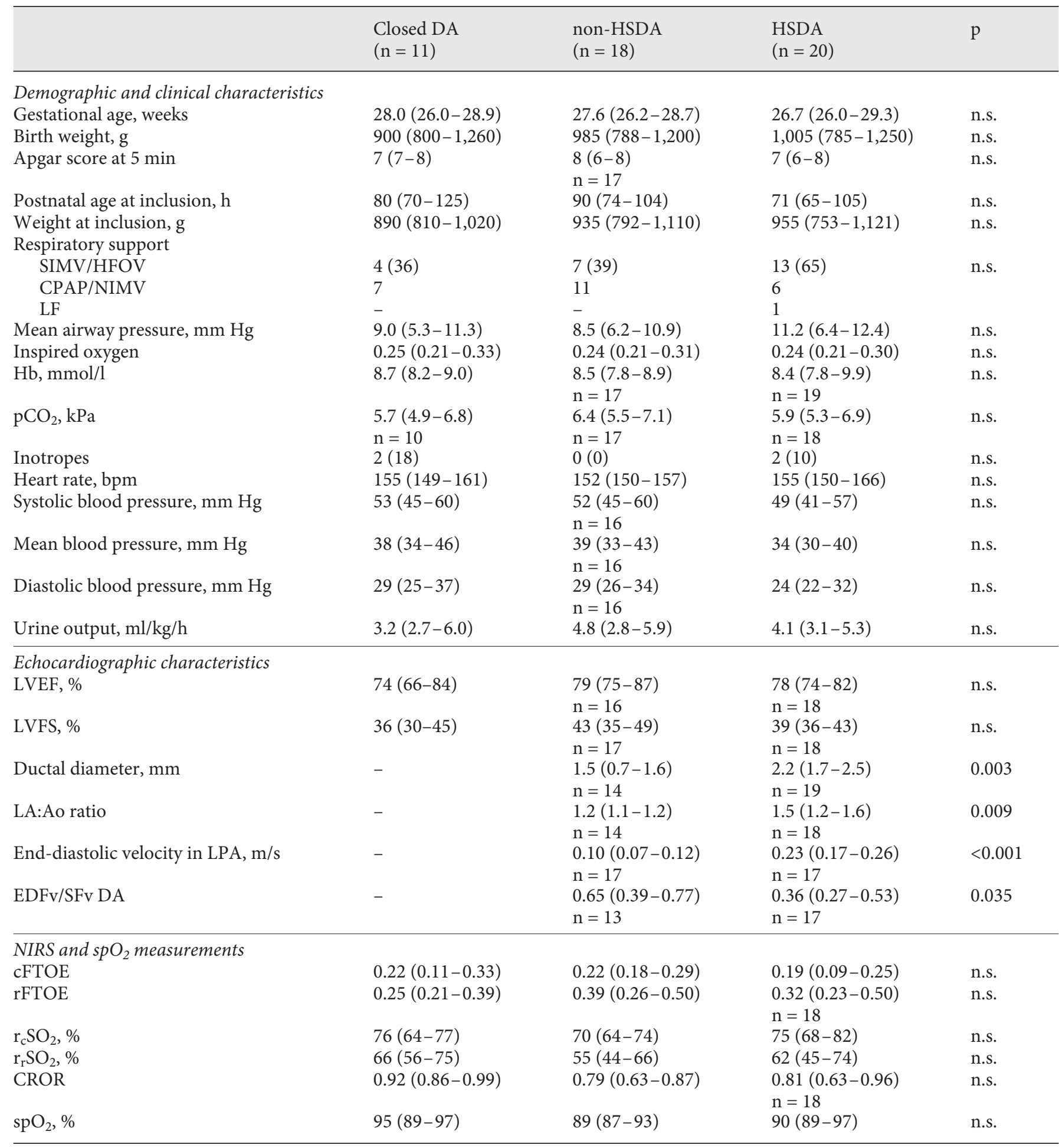

Data are presented as medians (IQR) or $\mathrm{n}(\%)$, as appropriate. SIMV = Synchronized intermittent mechanical ventilation; HFOV = high frequency oscillatory ventilation; $\mathrm{CPAP}$ = continuous positive airway pressure; NIMV = nasal intermittent mechanical ventilation; LF = low flow; LVEF = left ventricular ejection fraction; LVFS = left ventricular fractional shortening; EDFv = end-diastolic flow velocity; $\mathrm{SFv}=$ systolic flow velocity; cFTOE = cerebral FTOE; rFTOE = renal FTOE; n.s. = nonsignificant. 
13-15], we also calculated the cerebrorenal oxygenation ratio (CROR) as $\mathrm{CROR}=\mathrm{r}_{\mathrm{r}} \mathrm{SO}_{2} / \mathrm{r}_{\mathrm{c}} \mathrm{SO}_{2}$.

Mean 1-hour values of $\mathrm{r}_{\mathrm{c}} \mathrm{SO}_{2}, \mathrm{r}_{\mathrm{r}} \mathrm{SO}_{2}$, cerebral and renal FTOE, and CROR were calculated within $3 \mathrm{~h}$ preceding or following each echocardiogram, depending on the stability of the NIRS data. If NIRS parameters were not available within these $3 \mathrm{~h}$ and the echocardiogram showed a PDA, we did not include this particular infant in the present study. If the echocardiogram showed a closed $\mathrm{DA}$, we assumed that the DA remained closed for the next $24 \mathrm{~h}$ and included the infant if stable measurements during $1 \mathrm{~h}$ were available within $24 \mathrm{~h}$ following echocardiography. NIRS data were categorized as unreliable and excluded from analyses in the case of documented malplacement of sensors and in the case of baseline changes $>50 \%$ after the documented replacement of sensors.

\section{Demographic and Clinical Variables}

We prospectively collected clinical characteristics on the day of echocardiography including heart rate, blood pressure, urine output, $\mathrm{spO}_{2}, \mathrm{pCO}_{2}$, and hemoglobin levels. We also collected GA, birth weight, Apgar score, postnatal age, weight, ventilator mode and settings, and inotrope administration from the patients' medical charts.

\section{Statistical Analysis}

First, we classified all included infants into three groups: infants with a closed DA, infants with a non-HSDA, and infants with an HSDA. Second, we classified all infants with PDA into infants with retrograde diastolic blood flow in the Dao and infants without retrograde diastolic blood flow in the Dao. Differences in clinical characteristics, echo parameters, and NIRS measurements between groups were tested with the Kruskal-Wallis test and the MannWhitney test for continuous data and the $\chi^{2}$ test for categorical data, where appropriate. Spearman's correlation coefficients were calculated to assess the association between echo parameters of ductal hemodynamic significance and NIRS measurements in all infants with PDA. SPSS 22.0 (IBM Corp., Armonk, N.Y., USA) was used for all statistical analyses. A p value $<0.05$ was considered significant.

\section{Results}

We enrolled 57 infants, of whom 8 were excluded from further analysis due to poor NIRS signal integrity $(n=4)$, inability to reliably classify the hemodynamic significance of the DA due to incomplete echocardiography $(\mathrm{n}=3)$, and the presence of a ventricular septum defect $(\mathrm{n}=1)$. The remaining 49 infants had a median GA of 27.6 weeks (IQR: 26.1-29.0), a median birth weight of 980 g (IQR: 800-1,200), and a median postnatal age at the time of echocardiography of $77 \mathrm{~h}$ (IQR: 70-107). Patient and clinical characteristics of the 8 excluded infants did not differ from those of the study group.

Eleven infants had a closed DA. Of the 38 infants with PDA, 20 had an HSDA. Demographic and clinical characteristics did not differ between the three groups (table 1). Echocardiographic characteristics are also presented. Ducts were larger in infants with an HSDA compared with infants with a non-HSDA: median $2.2 \mathrm{~mm}$ (IQR: 1.7-2.5) versus $1.5 \mathrm{~mm}$ (IQR: $0.7-1.6$ ), $\mathrm{p}=0.003$. NIRS measurements did not differ between the three groups (table 1).

\section{Retrograde Diastolic Blood Flow in the Dao}

Of the 38 infants with PDA, echocardiography revealed retrograde diastolic blood flow in the Dao in 11 , whereas this was absent in 21 infants; in 6 infants, diastolic blood flow in the Dao could not reliably be evaluated due to poor signal discrimination. Clinical and patient characteristics did not differ between groups (table 2). Echocardiographic characteristics are also presented. NIRS measurements did not differ between infants with and infants without retrograde diastolic blood flow in the Dao (table 2).

\section{Correlation between Echocardiographic Parameters and NIRS Measurements}

Spearman's correlation coefficients between echocardiographic parameters of hemodynamic significance and NIRS measurements are presented in table 3. End-diastolic velocity in the LPA correlated significantly with renal FTOE (rho: $-0.418, \mathrm{p}=0.017$ ) and $\mathrm{rSO}_{2}$ (rho: 0.438, $\mathrm{p}=0.012$ ), but not with cerebral NIRS measurements.

\section{Discussion}

This study demonstrated that, in preterm infants with clinical signs of PDA during the first 2 weeks after birth, cerebral and renal oxygen saturation and extraction were not affected by the presence of a PDA, whether or not it was echocardiographically classified as hemodynamically significant. Furthermore, we demonstrated that in the presence of a PDA, cerebral and renal $\mathrm{rSO}_{2}$ and FTOE were not compromised by retrograde diastolic blood flow in the Dao. Other echocardiographic signs suggestive of an HSDA, such as increased ductal diameter, increased end-diastolic blood flow velocity in the LPA and increased LA:Ao ratio, were also not associated with impaired cerebral or renal NIRS measurements.

Echocardiographic signs used to classify a PDA as hemodynamically significant or not have previously all been related to large transductal shunt volumes [6, 7]. Still, we found no effect of the presence of an HSDA on cerebral or renal $\mathrm{rSO}_{2}$ and FTOE, and no significant associations between independent echocardiographic signs suggesting an HSDA and impaired cerebral and renal oxygen saturation and extraction. Our results suggest that, in the presence of an echocardiographically determined HSDA, 
Table 2. Demographic, clinical and echocardiographic characteristics of infants with and without retrograde diastolic blood flow in the Dao, and NIRS and $\mathrm{spO}_{2}$ measurements

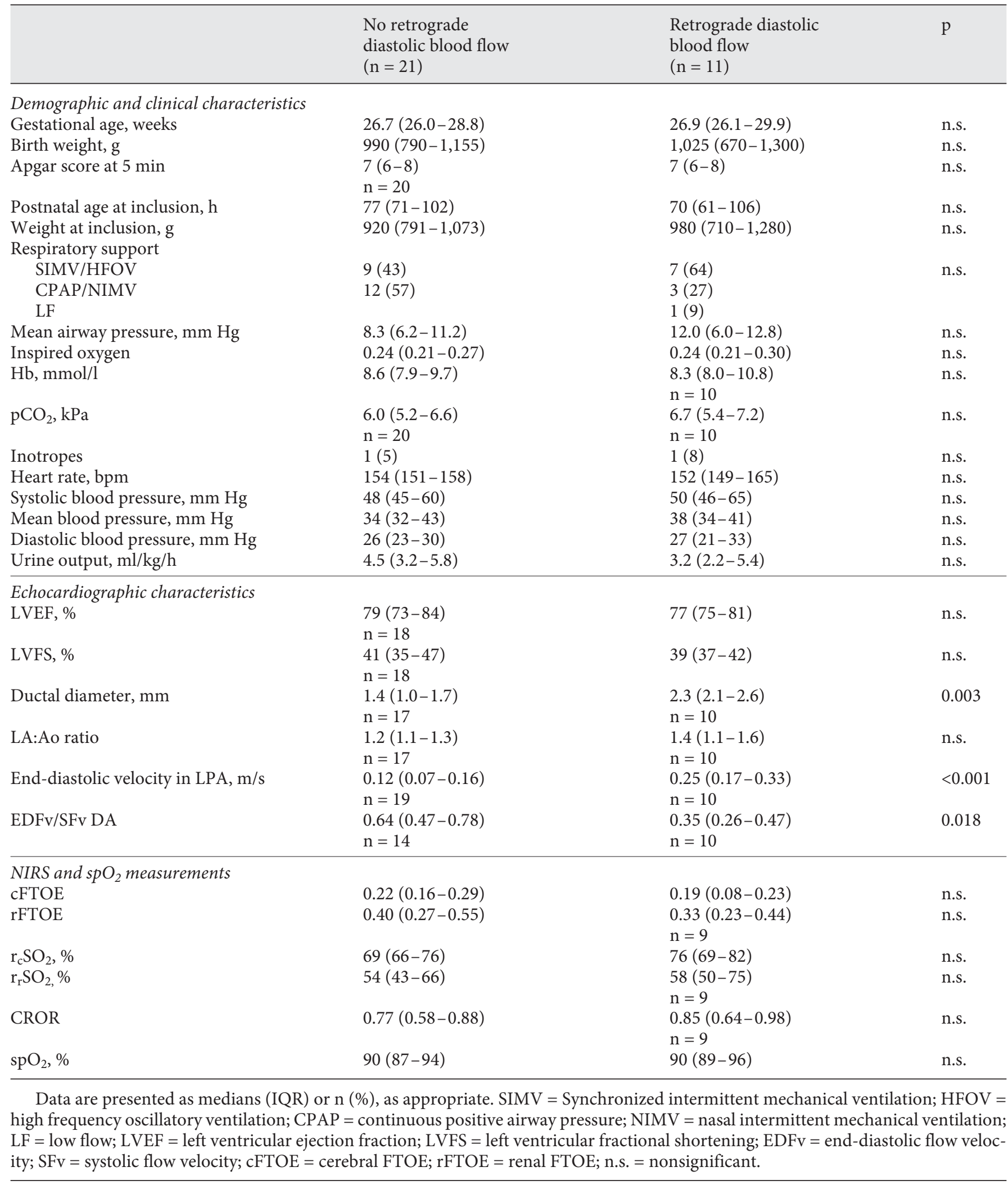


Table 3. Correlation between echo parameters and NIRS measurements in infants with PDA

\begin{tabular}{lrrrrr}
\hline & cFTOE & rFTOE & $\mathrm{r}_{\mathrm{c}} \mathrm{SO}_{2}$ & $\mathrm{r}_{\mathrm{r}} \mathrm{SO}_{2}$ & CROR \\
\hline Ductal diameter (mm) & -0.278 & -0.083 & 0.260 & 0.082 & -0.040 \\
LA:Ao ratio & -0.106 & -0.064 & 0.052 & 0.034 & 0.026 \\
End-diastolic velocity & & & & & \\
$\quad$ in LPA (m/s) & -0.181 & $-0.418^{*}$ & 0.293 & $0.438^{*}$ & 0.315 \\
EDFv/SFv DA & 0.252 & 0.097 & -0.249 & -0.098 & 0.009 \\
\hline
\end{tabular}

Data are presented as Spearman's rank order correlation coefficients. $\mathrm{cFTOE}=$ Cerebral FTOE; $\mathrm{rFTOE}=$ renal FTOE; $\mathrm{EDFv}=$ end-diastolic flow velocity; $\mathrm{SFv}=$ systolic flow velocity. ${ }^{*} \mathrm{p}<0.05$.

cerebral and renal oxygen delivery is not compromised. Our results are in line with previous studies reporting similar cerebral [9] and renal [10] $\mathrm{rSO}_{2}$ and FTOE in preterm infants with an HSDA compared with infants with a closed DA. They are also in line with a study reporting similar cerebral and renal $\mathrm{rSO}_{2}$ and FTOE in infants with large-sized ducts compared with infants with moderatesized ducts [11].

Conversely, Lemmers et al. [12] reported decreased cerebral $\mathrm{rSO}_{2}$ in infants with an HSDA compared with matched controls without clinical signs of a PDA. In this study, however, infants with an HSDA received inotropes more often than controls. Dopamine administration has previously been associated with impaired cerebrovascular autoregulation in preterm infants [16] and a mismatch between cerebral perfusion and oxygenation in newborn piglets [17]. We speculate that the higher rate of administration of dopamine might have contributed to their findings of decreased cerebral $\mathrm{rSO}_{2}$ in the presence of an HSDA, in contrast to all previous reports. In our cohort, only few infants received blood pressure support using inotropes.

In preterm infants with an HSDA, alterations in diastolic blood flow in several major arteries have been described, including the Dao, middle cerebral artery, renal artery, and superior mesenteric artery $[15,18]$. The relationship, however, between these echocardiographic markers of ductal steal, actual end-organ tissue oxygen delivery, and adverse outcome remains unclear [19]. Groves et al. [13] reported preserved superior vena cava flow but decreased Dao flow in preterm infants with PDA and retrograde diastolic blood flow in the Dao compared with infants with PDA without retrograde diastolic blood flow in the Dao during the first $48 \mathrm{~h}$ after birth. We did not find an effect of retrograde diastolic blood flow on cerebral or renal oxygen saturation and extraction. The results of the current study suggest that, at least on group level, cerebral as well as renal tissue oxygen delivery are preserved in the presence of retrograde diastolic blood flow in the Dao in preterm infants with postnatal age $>48$ h during the first 2 weeks after birth.

We recognize several limitations of our study. First, as we only included infants who underwent echocardiography mostly after the transitional period, but not after the first 2 weeks after birth, our results cannot be extrapolated to younger or older infants. Second, a failure to find any differences between groups might be due to the criteria for HSDA that we used, which are not generally agreed upon. Third, concerns have been raised concerning the accuracy and precision of cerebral NIRS monitoring. Previous studies have described a high replacement variability with limits of agreement of up to $18 \%$ [20-22]. Furthermore, while cerebral oximetry has extensively been studied, this is less true for renal oximetry. In our center, repeatability of $\mathrm{r}_{\mathrm{c}} \mathrm{SO}_{2}$ measurements revealed a mean difference of $4.5 \%$ (range 1-8) between three consecutive measurements of the same patient [Verhagen and Bos, unpubl. data]. Still, subtle associations between an HSDA and $\mathrm{rSO}_{2}$ might not have been detected.

\section{Conclusions}

The results of our study suggest that an HSDA and retrograde diastolic blood flow in the Dao do not have an adverse effect on cerebral and renal oxygen delivery in preterm infants who develop clinical signs of an HSDA during the first 2 weeks after birth.

\section{Acknowledgments}

We would like to thank A.J. Olthuis for helping with the data collection. This study was part of the research program of the Graduate School of Medical Sciences, Research Institutes BCNBRAIN and GUIDE, University of Groningen. M.E. van der Laan, T.E. Schat, A.G.J.F. van Zoonen, and J.C. Tanis were financially supported by a grant from the Junior Scientific Master Class of the University of Groningen. This study was funded by the Research Foundation of the Beatrix Children's Hospital and the 'Doelmatigheidsfonds' (a cost-effectiveness fund) of the University Medical Center Groningen.

\section{Disclosure Statement}

The authors declare that they have no conflicts of interest to disclose, financially or otherwise. van der Laan et al. 


\section{References}

1 Clyman RI: Mechanisms regulating the ductus arteriosus. Biol Neonate 2006;89:330-335.

2 Dollberg S, Lusky A, Reichman B: Patent ductus arteriosus, indomethacin and necrotizing enterocolitis in very low birth weight infants: a population-based study. J Pediatr Gastroenterol Nutr 2005;40:184-186.

3 Evans N, Kluckow M: Early ductal shunting and intraventricular haemorrhage in ventilated preterm infants. Arch Dis Child Fetal Neonatal Ed 1996;75:F183-F186.

4 Chorne N, Leonard C, Piecuch R, Clyman RI: Patent ductus arteriosus and its treatment as risk factors for neonatal and neurodevelopmental morbidity. Pediatrics 2007;119:11651174.

5 Sehgal A, McNamara PJ: Does echocardiography facilitate determination of hemodynamic significance attributable to the ductus arteriosus? Eur J Pediatr 2009;168:907-914.

6 El Hajjar M, Vaksmann G, Rakza T, Kongolo F, Storme L: Severity of the ductal shunt: a comparison of different markers. Arch Dis Child Fetal Neonatal Ed 2005;90:F419-F422.

7 Broadhouse KM, Price AN, Durighel G, Cox DJ, Finnemore AE, Edwards AD, Hajnal JV, Groves AM: Assessment of PDA shunt and systemic blood flow in newborns using cardiac MRI. NMR Biomed 2013;26:1135-1141.

8 Naulaers G, Meyns B, Miserez M, Leunens V, Van Huffel S, Casaer P, Weindling M, Devlieger $\mathrm{H}$ : Use of tissue oxygenation index and fractional tissue oxygen extraction as non-invasive parameters for cerebral oxygenation. A validation study in piglets. Neonatology 2007; 92:120-126
9 Chock VY, Ramamoorthy C, Van Meurs KP: Cerebral oxygenation during different treatment strategies for a patent ductus arteriosus. Neonatology 2011;100:233-240.

10 Guzoglu N, Sari FN, Ozdemir R, Oguz SS, Uras N, Altug N, Dilmen U: Renal and mesenteric tissue oxygenation in preterm infants treated with oral ibuprofen. J Matern Fetal Neonatal Med 2014;27:197-203.

11 Petrova A, Bhatt M, Mehta R: Regional tissue oxygenation in preterm born infants in association with echocardiographically significant patent ductus arteriosus. J Perinatol 2011;31:460-464.

12 Lemmers PM, Toet MC, van Bel F: Impact of patent ductus arteriosus and subsequent therapy with indomethacin on cerebral oxygenation in preterm infants. Pediatrics 2008;121: 142-147.

13 Groves AM, Kuschel CA, Knight DB, Skinner JR: Does retrograde diastolic flow in the descending aorta signify impaired systemic perfusion in preterm infants? Pediatr Res 2008; 63:89-94.

14 Hoodbhoy SA, Cutting HA, Seddon JA, Campbell ME: Cerebral and splanchnic hemodynamics after duct ligation in very low birth weight infants. J Pediatr 2009;154:196200 .
15 Shimada S, Kasai T, Hoshi A, Murata A, Chida S: Cardiocirculatory effects of patent ductus arteriosus in extremely low-birth-weight infants with respiratory distress syndrome. Pediatr Int 2003;45:255-262.

16 Eriksen VR, Hahn GH, Greisen G: Dopamine therapy is associated with impaired cerebral autoregulation in preterm infants. Acta Paediatr 2014;103:1221-1226.

17 Hahn GH, Hyttel-Sorensen S, Petersen SM, Pryds O, Greisen G: Cerebral effects of commonly used vasopressor-inotropes: a study in newborn piglets. PLoS One 2013;8:e63069.

18 Swarup J, Baker RW, Brozanski BS, Yanowitz TD: Asymmetry of cerebral blood flow velocity in low birth weight infants. Biol Neonate 2005;87:145-151.

19 Sehgal A, Coombs P, Tan K, McNamara PJ: Spectral Doppler waveforms in systemic arteries and physiological significance of a patent ductus arteriosus. J Perinatol 2011;31: 150-156.

20 Sorensen LC, Greisen G: Precision of measurement of cerebral tissue oxygenation index using near-infrared spectroscopy in preterm neonates. J Biomed Opt 2006;11:054005.

21 Dullenkopf A, Kolarova A, Schulz G, Frey B, Baenziger O, Weiss M: Reproducibility of cerebral oxygenation measurement in neonates and infants in the clinical setting using the NIRO 300 oximeter. Pediatr Crit Care Med 2005;6:344-347.

22 Wijbenga RG, Lemmers PM, van Bel F: Cerebral oxygenation during the first days of life in preterm and term neonates: differences between different brain regions. Pediatr Res 2011;70:389-394. 